\title{
The 8th World Workshop on Oral Health and Disease in AIDS
}

\section{David Croser reports on the WW8 event held in Indonesia in September}

Why was such a meeting thought necessary? After all, we live in an era with effective anti-retroviral therapy (ART) that has the potential to make a patient's viral load undetectable, and effective public health campaigns that have encouraged those at risk to get tested so that appropriate support and medication can be provided for anyone found to be HIV positive.

A similar scenario sits behind the targets set by the joint United Nations programme on HIV/AIDS (UNAIDS). UNAIDS challenged nations to diagnose $90 \%$ of all HIV-positive persons, provide ART for $90 \%$ of those diagnosed, and achieve viral suppression for $90 \%$ of those treated by 2020 with the ultimate aim of ending the AIDS epidemic by 2030.

Whilst the UK continues to move towards the 2020 target, things in Indonesia are quite different. The situation there resembles that which we were facing in Europe and North America several decades ago. It was for this reason that the Indonesian Dental Association and the International Association for Dental Research were keen to convene the eighth World Workshop (WW8) in Indonevsia (population 262 million).

Six hundred and forty delegates attended WW8 from 36 different countries to share the collective experience of dentists and doctors, together with specialists in oral medicine and public health - all of whom have an interest in HIV/AIDS.

In Indonesia it is estimated that $50 \%$ of people carrying the disease do not know their HIV status; less than $20 \%$ of those diagnosed are on ART; and many of those on ART are not immunosuppressed because compliance is poor.

The meeting was chaired by Professor Anwar Tappuni (Queen Mary University of London) and was ably supported by the organisational skills of Professor Irna Sufiawati (University of Padjadjaran, West Java). WW8 had excellent material on global epidemiology, social sciences, testing, prevention, treatment and much outstanding basic science. During the sessions it became clear that there was a need for education, for

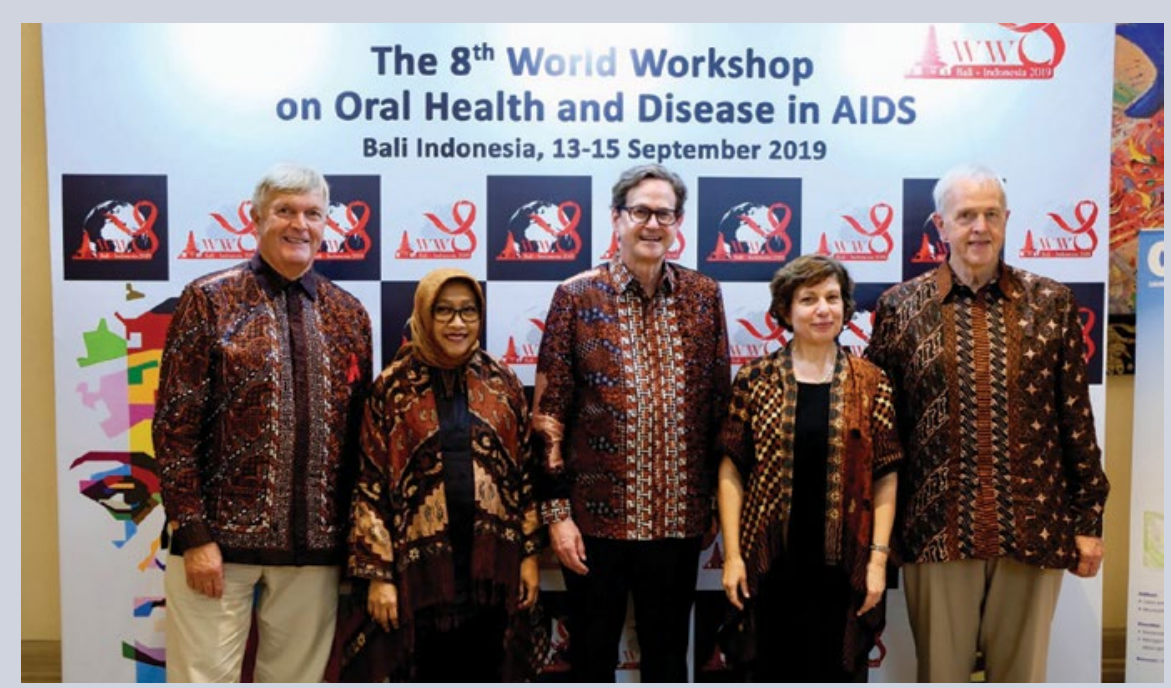

Representing the conference organisers. Left to right: Dr David Croser (UK), Prof Irna Sufiawati (Java), Dr Christopher Hughes Fox (IADR, USA), Prof Anwar Tappuni (UK), Prof Stephen Challacombe (UK)

both the population and the medical professions, to stem the tide and the essential role of the oral health professions therein.

Each of the former World Workshops has produced a declaration. All have been influential, particularly that from the Beijing meeting in 2009: this influenced the UK (and others nations since) to revise the policy on permitting HIV positive dentists to continue clinical practice - with conditions based on scientific evidence rather than emotion. The latest Workshop was no different and a consensus view of delegates was voted on during the final session and carried nem. con.

An international consensus view based on peer-reviewed research is a powerful voice to add to the voices of the many Indonesian dentists and dental students in training in their effort to end the AIDS epidemic by 2030 . The trusted voice of dental professionals can also make a difference in ending the epidemic in whichever country they live.

Having reviewed the evidence presented to the WW8, the delegates agreed that the UNAIDS objective of ending the global HIV/ AIDS epidemic by 2030 could be significantly enhanced by improving the current level of knowledge in healthcare professionals and the public at large. The workshop concluded that it is necessary to eliminate the stigma of HIV/ AIDS, and to improve accessibility and compliance with effective therapy in order to stop HIV transmission. Targeted educational input is required to ensure: that the role of dental professionals is recognised as an integral part of the healthcare team committed to achieving the aims of UNAIDS; that HIV education of dental students and dental healthcare workers should be an essential element of the dental curriculum to ensure the profession has the appropriate knowledge and attitudes to manage people living with $\mathrm{HIV}$; and that public health programmes make certain that the general public is aware of HIV testing and the role of dental healthcare workers in facilitating this: thereby further normalising attitudes to people living with HIV.

Research into oral health serves to improve the diagnosis and care of patients and provides the evidence base that underpins future education. High quality research and educational initiatives for doctors, dentists and the community are urgently required to achieve the UNAIDS objectives.

\section{Useful links}

- 8th World Workshop on Oral Health in HIV/AIDS: www.ww8aids.org

- UNAIDS targets: www.unaids.org

- Effect of Beijing Declaration in UK: Croser D. Welcome news. Br Dent J 2013; 215: 201-202. 